\title{
Rehabilitación protésica en paciente pediátrico con displasia ectodérmica
}

\section{Resumen}

La displasia ectodérmica es un trastorno congénito caracterizado por el desarrollo anormal de dos o más derivados de ectodermo, como el cabello, glándulas sudoríparas, uñas y dientes. Los tipos más comunes son la Hipohidrótica/ Anhidrótica y la Hidrótica. El objetivo del reporte de caso es mostrar las características de un paciente con displasia ectodérmica anhidrótica, el tratamiento odontológico realizado y los resultados logrados. Reporte de caso: Paciente masculino de 5 años con Displasia Ectodérmica Anhidrótica acude en compañía de su madre a la clínica de especialidad de Odontopediatría de la ENES UNAM León, quien reporta como motivo de consulta: "que le coloquen dientes". En la exploración extraoral se observa cabello escaso y fino, ausencia de cejas y pestañas, proquelia, tercio inferior disminuido, en la evaluación intraoral
Jessica Vianey Aparicio Muñoz, ${ }^{1}$

Tatiana Dinhora Mondragón Báez, ${ }^{1}$ Rogelio Danovan Venegas Lancón, ${ }^{1}$ se observa oligodoncia con presencia de dientes cónicos en 52 y 62, en la ortopantomografía se observa en proceso de erupción los dientes 11 y 21. Diagnóstico bucal: Oligodoncia, dientes cónicos, retardo de la erupción, reborde alveolar atrófico-estrecho y disminución de la dimensión vertical. Tratamiento: Rehabilitación de coronas de disilicato de litio en los dientes 52 y 62, prótesis parcial removible con tornillo de expansión en el maxilar superior y, en la mandíbula, prótesis total con tornillo de expansión. Se generó una mejor función masticatoria, fonación, peristaltismo lingual y mayor autoestima del paciente. Conclusiones: La rehabilitación temprana en pacientes con Displasia Ectodérmica y su tratamiento integral, promueve el crecimiento de las estructuras bucales, función, estética y autoestima del paciente.

Palabras clave: Displasia ectodérmica, oligodoncia, prostodoncia, odontopediatría. 
Caso clinico

\section{Reabilitação protética em paciente pediátrico com displasia ectodérmica}

\section{Resumo}

A displasia ectodérmica é um distúrbio congênito caracterizado pelo desenvolvimento anormal de dois ou mais derivados do ectoderma, como cabelos, glândulas sudoríparas, unhas e dentes. Os tipos mais comuns são Hipohidrótico / Anidrótico e Hidrótico. O objetivo do relato de caso é mostrar as características de um paciente com Displasia Ectodérmica anidrótica, bem como o tratamento odontológico realizado e os resultados alcançados. Relato de caso: Paciente do sexo masculino, 5 anos, portador de Displasia Ectodérmica Anidrótica, vai com sua mãe à Clínica de Odontopediatria da ENES UNAM León, quem relata como motivo da consulta "Colocar os dentes". No exame extra-oral, são observados cabelos finos e esparsos, ausência de sobrancelhas e cílios, terço inferior diminuído, no exame intraoral, oligodontia com presença de dentes cônicos 52 e 62, na radiografia panorâmica mostra os dentes 11 e 21 . Diagnóstico odontológico: Oligodontia, dentes cônicos, erupção tardia, rebordo alveolar atrófico estreito e diminuição da dimensão vertical. Tratamento: Reabilitação de coroas de dissilicato de lítio nos dentes 52 e 62 , prótese parcial removível com parafuso de expansão na maxilar superior e prótese total com parafuso de expansão na mandíbula. Geraram melhor função mastigatória, fonação, peristaltismo lingual e maior autoestima do paciente. Conclusões: A reabilitação precoce em pacientes com displasia ectodérmica e seu tratamento abrangente promove o crescimento das estruturas orais, função, estética e auto-estima do paciente.

Palavras-chave: Displasia ectodérmica, oligodontia, prótese dentária, odontopediatria.

Case report

\section{Prosthetic rehabilitation in pediatric patient with ectodermal dysplasia}

\begin{abstract}
Ectodermal dysplasia is a congenital disorder characterized by the abnormal development of two or more ectoderm derivatives, such as hair, sweat glands, nails and teeth. The most common types are Hypohydrotic / Anhydrotic and Hydrotic. The objective of the case report is to show the characteristics of a patient with Ectodermal Anhydrotic Dysplasia,
\end{abstract}

as well as the dental treatment performed and the results achieved. Case Report: A 5-year-old male patient with ectodermal anhydrotic dysplasia attends with his mother to the Pediatric dentistry clinic at ENES UNAM León, who reports as a reason for consultation "of having teeth placed". In the extraoral exam, there is an evident lack of eyebrows and eyelashes, thin hair, prochelia and decreased lower facial third is observed. Within 
the intraoral evaluation, oligodontia is observed along with a conical shape of teeth 52 and 62. In the panoramic radiography, the presence of teeth 11 and 21 is observed. Dental diagnosis: Oligodontia, conical teeth, delayed eruption, narrow atrophic alveolar rim, and decreased vertical dimension. Treatment: Rehabilitation with a removable partial prosthesis with expansion screw in the upper jaw along with lithium disilicate crowns on teeth 52 and 62, and

\section{Introducción}

La displasia ectodérmica (DE) es un trastorno congénito caracterizado por el desarrollo anormal de dos o más derivados de ectodermo. ${ }^{1}$ Caracterizado por alteraciones en el cabello y vello corporal, glándulas sudoríparas, uñas, dientes, ojos, dedos de las manos y pies, entre otras partes del cuerpo., , $^{2} \mathrm{Su}$ prevalencia va a variar de entre 1:10,000 a 1:100,000 nacidos vivos, ${ }^{4}$ tiene mayor prevalencia en hombres y los casos son más severos. ${ }^{5}$

$\mathrm{Su}$ fenotipo es una expresión genética ya sea autosómica recesiva, autosómica dominante o ligada al cromosoma X. La displasia ectodérmica hidrótica tiene un patrón autosómico dominante y es menos común. La displasia ectodérmica hipohidrótica o anhidrótica tiene un patrón de herencia autosómico recesivo ligado al cromosoma $X$, constituyendo el $80 \%$ de los afectados. Sin embargo, puede presentarse sin historial familiar previo debido a una mutación del gen Xq12-q13. ${ }^{1,6}$

Existen más de 197 tipos de displasia ectodérmica, solo se conoce el gen causante en 60 de ellos. Las más comunes se dividen a complete denture prosthesis with an expansion screw in the lower jaw. A better masticatory function, phonation, lingual peristalsisand greater patient self-esteem were achived. Conclusion: Early rehabilitation and integral treatment promotes the growth of the oral structures, function, aesthetics and self-esteem of the patient.

Key words: Ectodermal dysplasia, oligodontia, dental prosthesis, pediatric dentistry.

en 2 grupos: Displasia ectodérmica hidrótica o Síndrome de Clouston se caracteriza por hipotricosis, distrofia ungueal e hiperqueratosis de las palmas de las manos y plantas de los pies. ${ }^{1,3,7,8}$ El segundo grupo incluye displasia ectodérmica anhidrótica e hipohidrótica (DEH), también conocida como Síndrome de Christ-Siemens-Touraine se caracteriza por la triada de hipotricosis, hipohidrosis (DE hipohidrótica) o anhidrosis (DE anhidrótica) e hipodoncia. . $^{3,8}$

Las características cráneo faciales que encontramos en estos pacientes es escaso cabello y vello corporal, piel seca e hipopigmentada, frente y mentón prominente, pigmentación de la piel alrededor de los ojos y la boca, mayor distancia intercantal, nariz en forma de silla de montar, depresión del tercio medio, dimensión vertical reducida, labios prominentes, hipoplasia del maxilar, protrusión mandibular, entre otras. ${ }^{1,2,9-11}$

En las manifestaciones orales podemos observar que la hipodoncia se da en un paciente con la ausencia de menos de seis dientes congénitamente, excluyendo al 3er. molar, oligodoncia se da en un paciente con seis o más dientes ausentes 
congénitamente, con excepción del 3er molar. La Anodoncia es la ausencia total congénita de todos los dientes. Afecta ambas denticiones, es una anomalía rara y cuando se produce, suele estar asociada con un trastorno más generalizado, como es la displasia ectodérmica hereditaria. La Anodoncia verdadera es la ausencia congénita de dientes se clasifica en: total o parcial. En la Anodoncia total, todos los dientes están ausentes, afectando tanto los de la dentición temporal como la permanente. La anodoncia parcial verdadera involucra uno o más dientes; crestas alveolares atróficas, afiladas y estrechas debido a la ausencia de dientes, alteraciones en el esmalte como hipoplasias, dientes cónicos, entre otros. ${ }^{1,9}$

El diagnóstico prenatal se puede realizar cuando existan antecedentes de familiares con displasia ectodérmica mediante análisis de ligamiento basado en ADN y pruebas genéticas para detectar mutaciones en el gen de ectodisplasina (EDA o EDA1) en el caso de DEH. La ultrasonografía tridimensional identificando las características faciales, la biopsia de piel fetal y el estudio genético de vellosidades coriónicas son pruebas de diagnóstico adecuadas en el segundo trimestre del embarazo. ${ }^{12,13}$

El diagnóstico debe ser evaluado en base a las características clínicas antes descritas. En el caso de la DEH o DEA puede reconocerse al nacimiento $\mathrm{o}$ a edades tempranas debido a los episodios de hiperpirexia, siendo esto muy peligroso, potencialmente mortal, se debe confirmar con las pruebas genéticas. El diagnóstico diferencial de la DEH o DEA puede incluir otros tipos de displasias como la displasia odonto-onico-dérmica y ciertas formas de ictiosis. $^{14,15}$
El tratamiento médico para la Displasia Ectodérmica lo realiza un equipo multidisciplinario de especialistas según las estructuras afectadas, atendiendo a cada una de sus características clínicas, por lo que es de vital importancia hacer hincapié en darle al paciente un tratamiento integral. ${ }^{15}$

El médico tratante debe estar muy al pendiente de los pacientes con $\mathrm{DEH}$ y DEA para disminuir la tasa de mortalidad, ya que estos pacientes sufren numerosas complicaciones, como retardo del crecimiento, infecciones pulmonares e hipertermia. Se debe proteger la exposición de altas temperaturas hidratando la piel y las mucosas, utilizar lágrimas artificiales para proteger la córnea, entre otros. ${ }^{12,13}$

Debido a las alteraciones físicas que presentan estos pacientes existe un deterioro en su desarrollo social y psicológico, afectando a su autoestima debido a que aparentan de una edad mayor, por la ausencia de dientes y pérdida de dimensión vertical, por lo que es de vital importancia iniciar el tratamiento odontológico desde edades tempranas..$^{6,8,9}$

Los tratamientos odontológicos dependerán de la edad del niño, su desarrollo dental y la severidad del caso debido a que las ausencias dentales tienen como consecuencia la hipoplasia maxilar y el colapso del tercio inferior de la cara. Es por ello que el Odontopediatra debe intervenir desde edades tempranas en estos pacientes y acompañar su crecimiento y desarrollo, con el uso de prótesis parciales o totales que promuevan la expansión transversal de los maxilares y la posible intervención de otras especialidades odontológicas que colaboren con mejorar la estética y función de estos pacientes. ${ }^{12}$ 
El tratamiento odontológico sugerido por la literatura es la rehabilitación protésica; con prótesis fija, parcial removible, prótesis total o prótesis implantosoportadas a partir de los 15 a 16 años. La prótesis removible es recomendada como tratamiento en niños para guiar el crecimiento y desarrollo cráneo facial, mejorar la función masticatoria, fonética, apariencia facial y una mayor autoestima del paciente. ${ }^{2,9}$

En casos de alteraciones de la forma dental en pacientes pediátricos la literatura recomienda las coronas de disilicato de litio que son cerámicas reforzadas, necesitan el mínimo degaste dental y proporcionan alta resistencia mecánica, estética y éxito a largo plazo. ${ }^{16}$

El objetivo del reporte de caso es mostrar las características de un paciente con displasia ectodérmica anhidrótica, así como el tratamiento dental realizado y los resultados logrados.

\section{Reporte de caso}

Asiste a consulta un paciente de género masculino de 5 años de edad, nacido en León, Guanajuato México el 21 de Junio del 2014 quien se presenta en compañía de madre a la clínica de Odontopediatría de la Escuela Nacional de Estudios Superiores UNAM Unidad León, Guanajuato México y reportando como motivo de consulta: "que le coloquen dientes".

En sus antecedentes familiares la madre reporta: "padecer displasia ectodérmica" y ser portadora de "molusco contagioso" diagnosticado a los 3 meses de gestación, la cual fue tratada con crioterapia.
En los antecedentes médicos del paciente la madre señala nacimiento por cesárea a las 39 semanas de gestación, con diagnóstico médico de Displasia Ectodérmica de tipo Anhidrótica a los 15 días de vida, a los 3 meses presentó crisis convulsiva y cirugía por obstrucción del píloro, a los 4 años presentó crisis febriles y anemia microcítica hipocrómica.

En los antecedentes odontológicos del paciente la madre señala no haber llevado a su hijo anteriormente al odontólogo.

Al examen clínico general se evidenció que presenta las siguientes manifestaciones físicas generales: hipotricosis, piel seca, ausencia de cejas y pestañas, hipertelorismo, piel periorbitaria hiperpigmentada, orejas prominentes y bajas. Al examen clínico extrabucal se evidenció en las características cráneo faciales implantación baja de pabellones auriculares, depresión del tercio medio facial, tercio inferior facial disminuido, labios gruesos, resecos e hipotónicos (Figura 1).

En el examen clínico intraoral se observa AnodonciaParcial Verdadera con presencia únicamente de los dientes 52 y 62 de forma cónica, reborde alveolar atrófico y estrecho y disminución de la dimensión vertical (Figura 2). En la ortopantomografía se observa la ausencia de todos los gérmenes dentarios permanentes con excepción de los dientes 11 y 21 en forma cónica (Figura 3) (Tabla 1).

En el tratamiento odontológico se realiza un plan de tratamiento integral, rehabilitación con coronas de Disilicato de litio en los dientes 52 y 62, prótesis parcial removible con tornillo de expansión en el maxilar superior, prótesis total con 

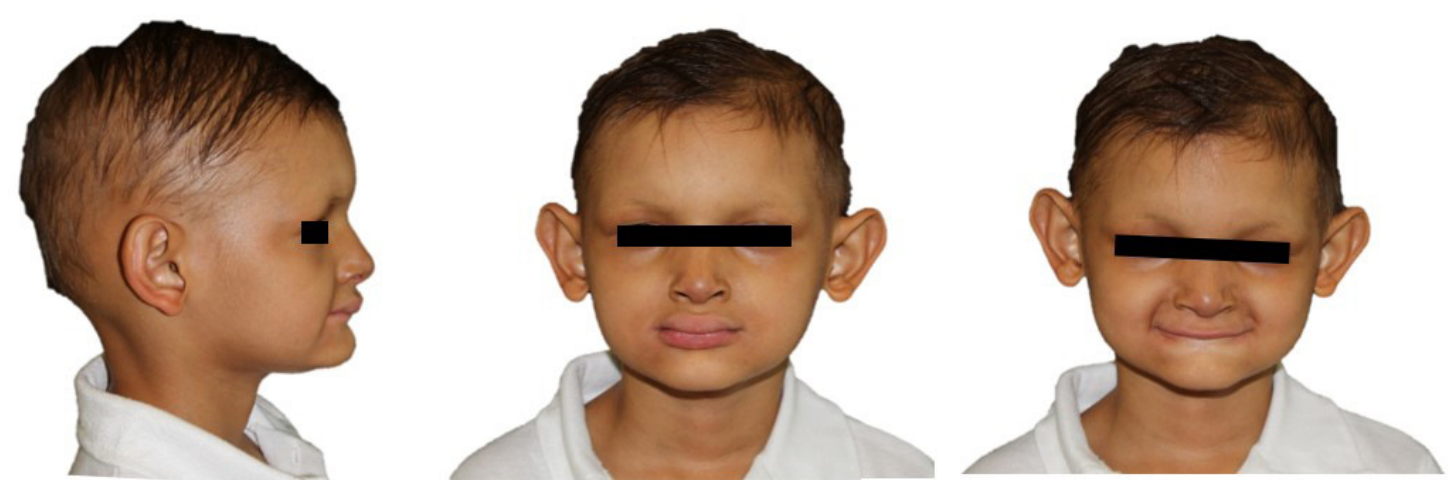

Figura 1. Fotografias extraorales iniciales.

tornillo de expansión en la mandíbula, interconsultas y referencias realizadas: Terapia de lenguaje para mejorar la fonación y ejercicios miofuncionales $\mathrm{y}$ de reposicionamiento de la lengua. Los controles periódicos para la aparatología mensualmente y controles odontológicos de acuerdo a su riesgo a caries cada 6 meses. El plan de tratamiento fue explicado tanto a los padres como al paciente. El caso fue sometido por el Comité de ética de la
Escuela Nacional de Estudios Superiores, UNAM, Unidad León; el cual fue aceptado para su realización (CEI_20_03_S3).

El tratamiento odontológico se inicia con impresiones de alginato (Tropicalgin de Zhermack), obtención de modelos y elaboración de cubetas individuales, posteriormente se realizó la impresión funcional con silicona (Body ExpressTM STD de 3M ESPE). Se obtuvo el modelo

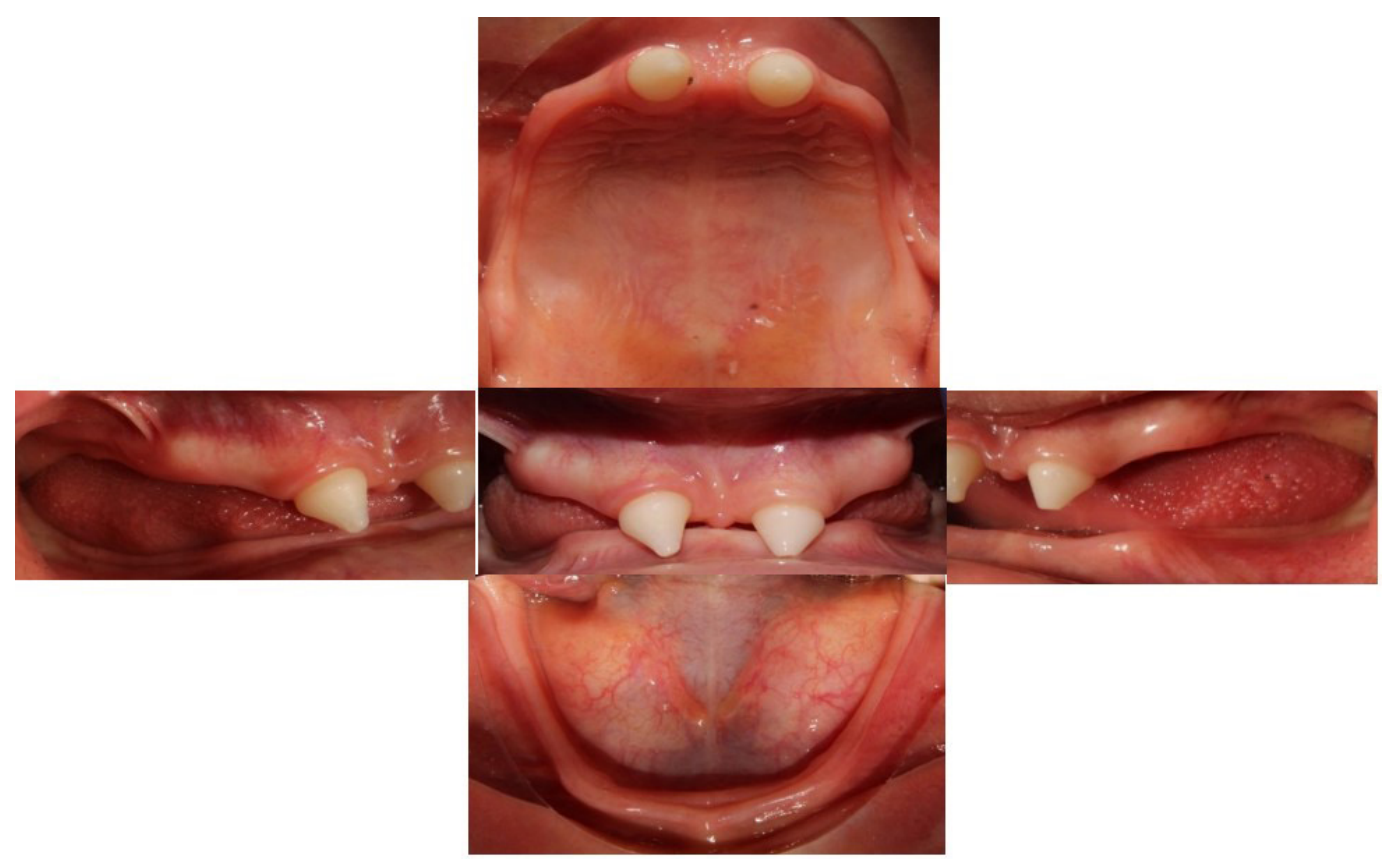

Figura 2. Fotografías intraorales iniciales. 


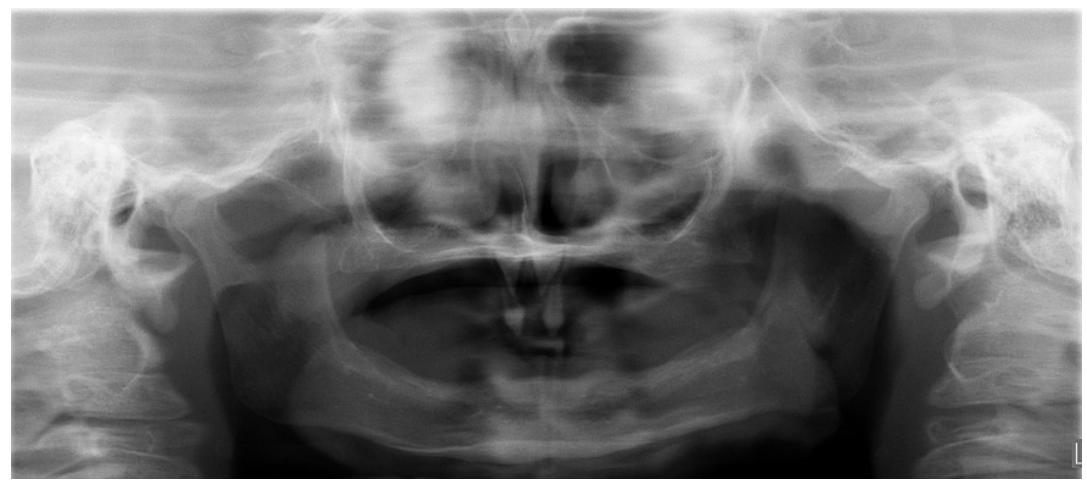

Figura 3: Radiografía panorámica.

de estudio sobre el que se elaboró la base con rodillos de cera, realizándose posteriormente las pruebas de ellas en el paciente (Figura 4) y se articuló en verticulador. Se realizó la articulación de dientes de acrílico anteriores y posteriores (Figura 5), se realizó prueba de dientes en el paciente. Posteriormente se colocaron los tornillos de expansión en la prótesis superior e inferior y se procesaron las prótesis en el Laboratorio de Prótesis Bucal Avanzada de la ENES León de la UNAM (Figura 6).
Se probaron las prótesis y se ajustaron (Figura 7), se tallaron los dientes 52 y 62 para mejorar la estética dental, realizando un desgaste de $0,5 \mathrm{~mm}$ en todas las caras para restaurarlos con coronas de disilicato de litio. Posteriormente, se tomaron impresiones con silicón ligero (Body Express TM STD de 3M ESPE) y se diseñó digitalmente y se fresaron en el sistema CAD-CAM del Laboratorio de Prótesis de la ENES León de la UNAM.

Una vez que el laboratorio nos entregó las coronas de disilicato de litio, se probaron

Tabla 1: Diagnóstico integral del paciente

\begin{tabular}{|l|l|}
\hline \multicolumn{2}{|c|}{ Diagnóstico } \\
\hline Genético & $\begin{array}{l}\text { Displasia ectodérmica } \\
\text { Anhidrótica }\end{array}$ \\
\hline Médicos & $\begin{array}{l}\text { Anhidrosis } \\
\text { Crisis febriles }\end{array}$ \\
\hline Odontológicos & $\begin{array}{l}\text { Alteraciones de forma (dientes cónicos) en 52 y 62. } \\
\text { Alteraciones de forma en 11 y 21 (hallazgo radiográfico). } \\
\text { Oligodoncia de dientes primarios: 55, 54, 53, 51, 61, 63, 64, 65, 75, 74, 73, 72, } \\
71,81,82,83,84,85 . \\
\text { Oligodoncia de dientes permanentes: 17, 16, 15, 14, 13, 12, 22, 23, 24, 25, 26, 27, } \\
\text { 37, 36, 35, 34, 33, 32, 31, 41, 42, 43, 44, 45, 46, 47. } \\
\text { Anodoncia parcial verdadera. } \\
\text { Maxilares atróficos. } \\
\text { Pérdida de la dimensión vertical. }\end{array}$ \\
\hline
\end{tabular}




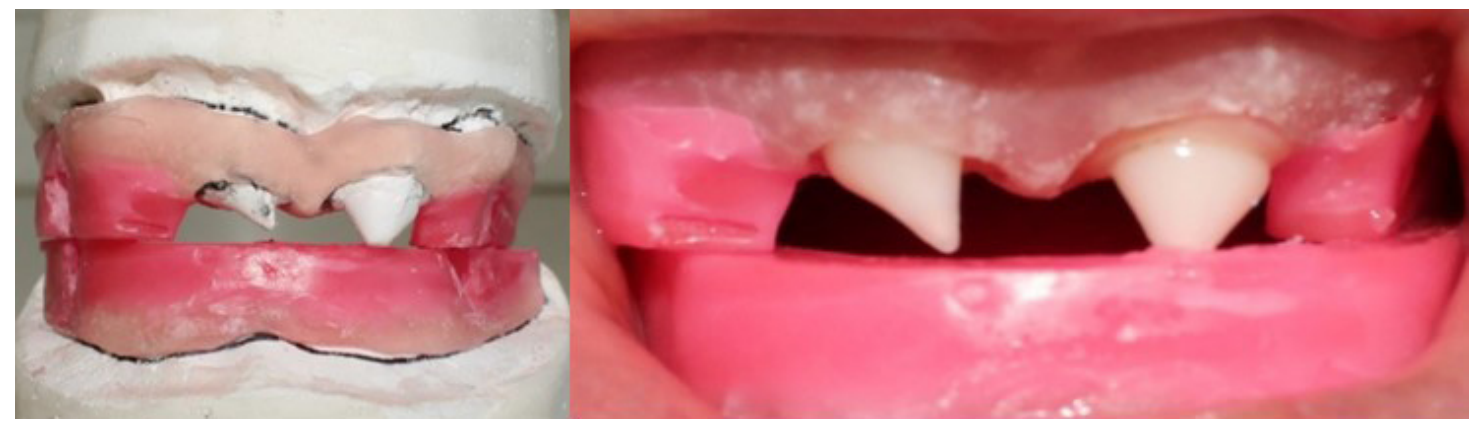

Figura 4: Prueba de rodillos de cera.

en boca y se acondicionaron los dientes y las coronas de acuerdo al protocolo establecido para coronas de disilicato de litio, en el que se debe acondicionar las coronas con ácido fluorhídrico (Porcelain Etch de Ultradent ${ }^{\circledR}$ ) por 20 segundos, posteriormente se enjuaga y se coloca ácido fosfórico al 35\% (Ultra-Etch de Ultradent ${ }^{\circledR}$ ) durante 1 minuto, se enjuaga nuevamente y se aplica silano (Silane de Ultradent ${ }^{\circledR}$ ) por 1 minuto; posteriormente se realiza profilaxis de los dientes y se acondicionan con ácido fosfórico al 35\% (Ultra-Etch de Ultradent $^{\circledR}$ ) durante 10 segundos, se lava $\mathrm{y}$ se coloca adhesivo (Futurabond NR de Voco), se realizó fotocurado por 20

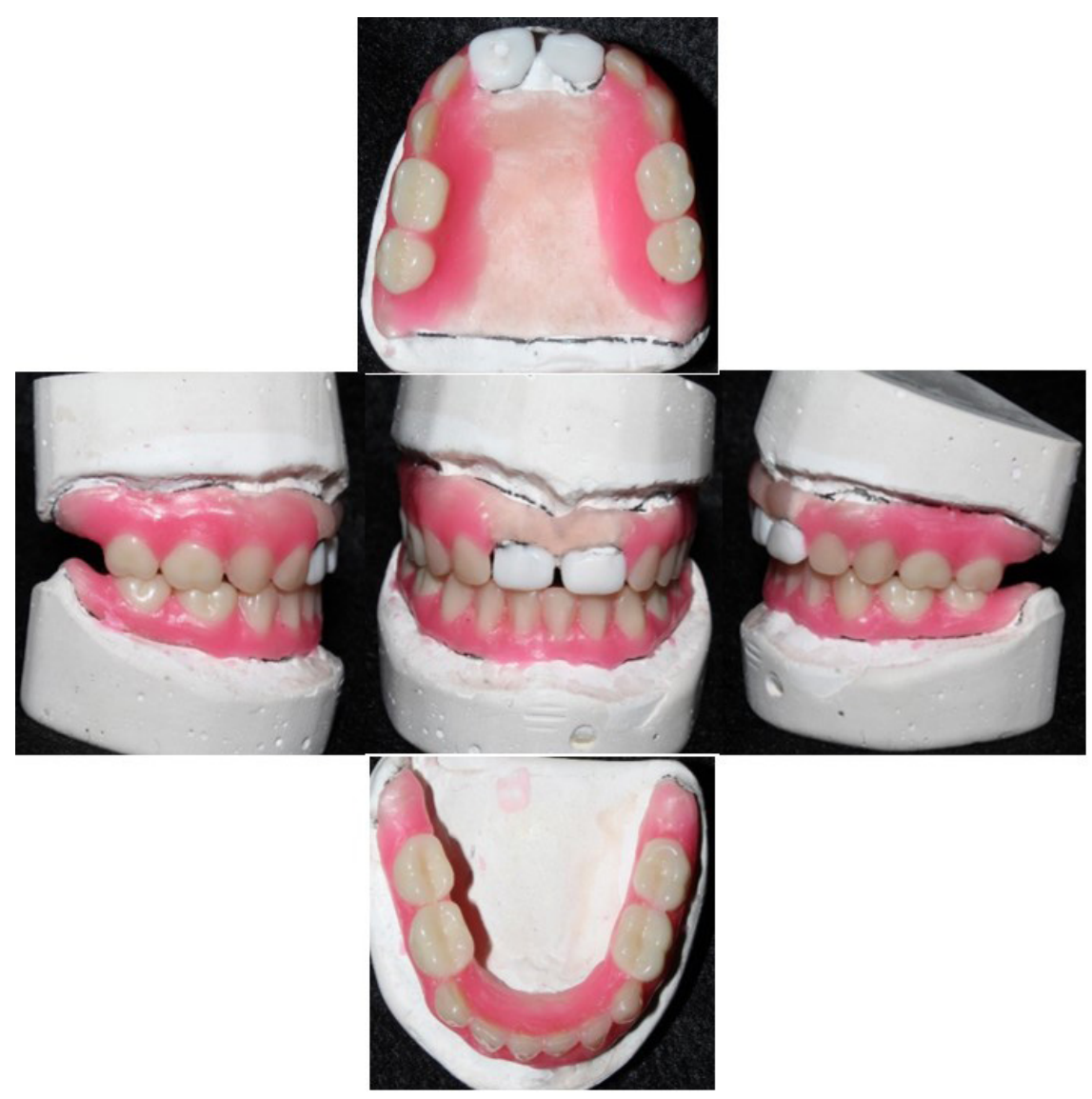

Figura 5: Colocación de dientes de acrílico. 


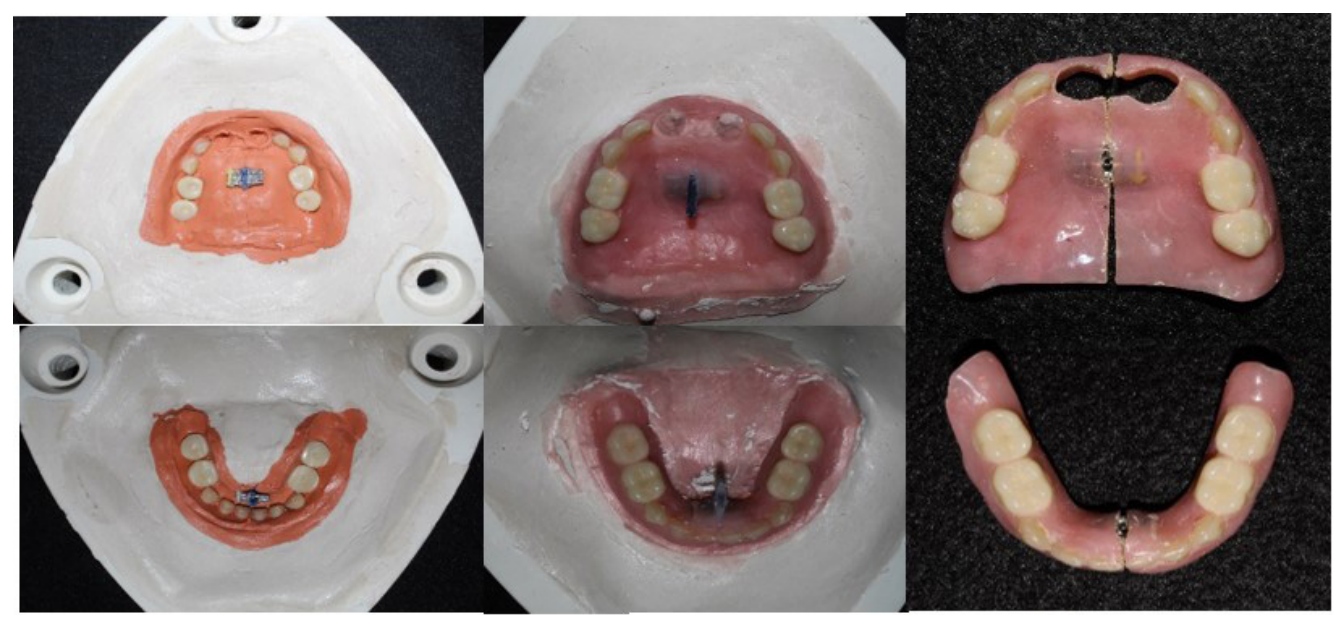

Figura 6: Procesado de prótesis removibles

segundos, se cementaron con cemento resinoso dual (Maxcem EliteTM de Kerr) y se fotocuró nuevamente durante 20 segundos por cada cara (Figura 8). Se le dieron indicaciones sobre los cuidados de las coronas. En cuanto a las prótesis removibles, se suministraron las indicaciones al paciente y a su madre acerca del cuidado de las prótesis, el uso de crema adhesiva para prótesis (Ultra Corega de GSK). Además, se indicó dar 1/4 de vuelta a la semana al tornillo de ambas prótesis para estimular el crecimiento y mejorar la conformación de ambas arcadas (Figura 10).

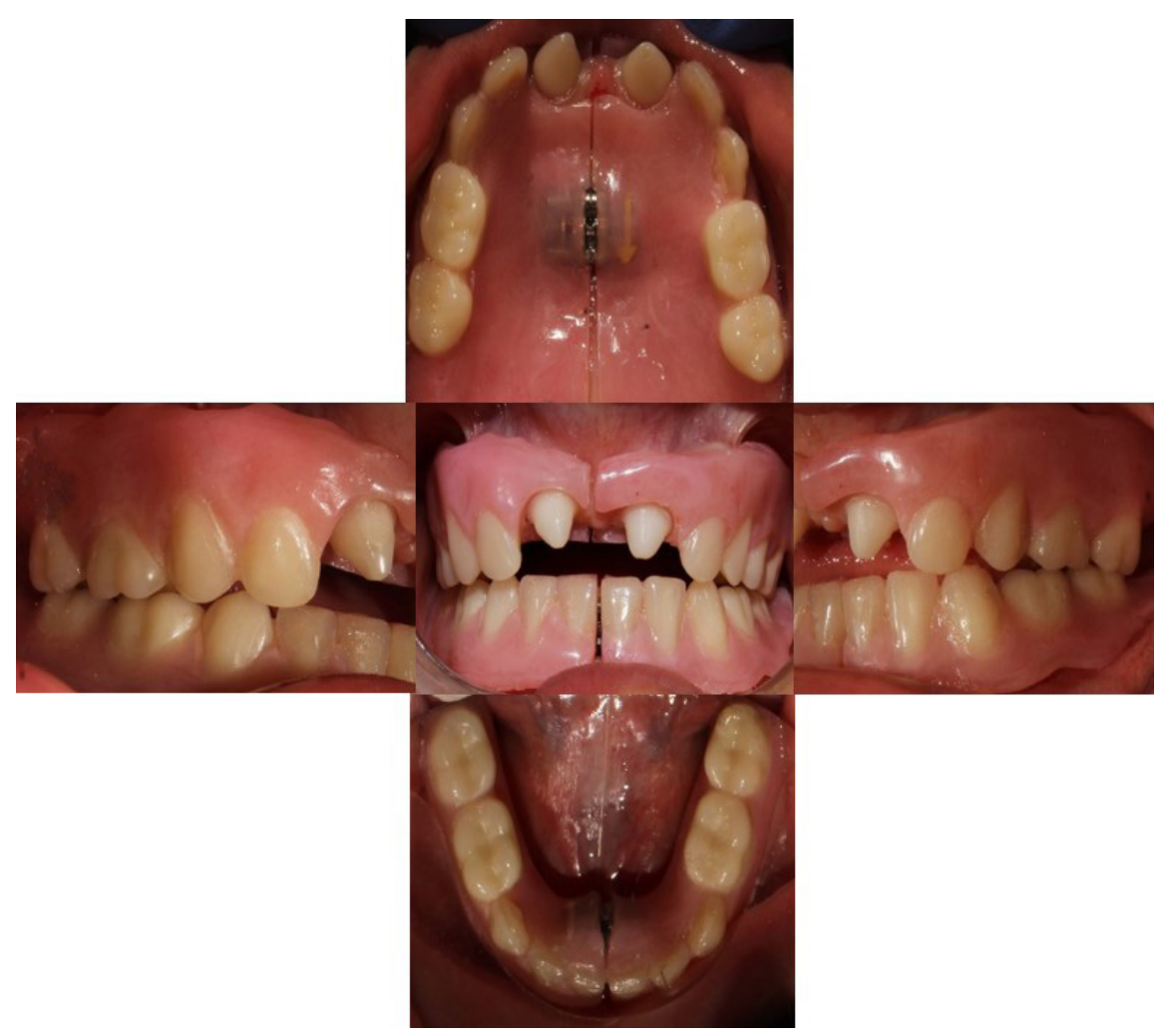

Figura 7: Colocación de prótesis removibles 


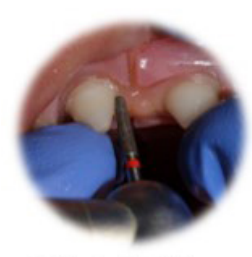

Tallado de $0.5 \mathrm{~mm}$

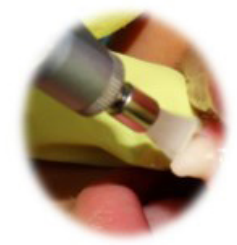

Cepillar los dientes

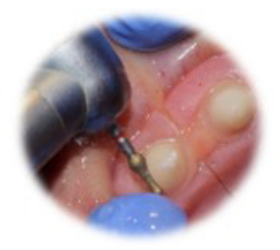

Redondeo de bordes

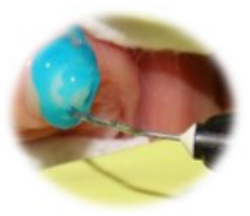

Ácido grabador $10 \mathrm{seg}$

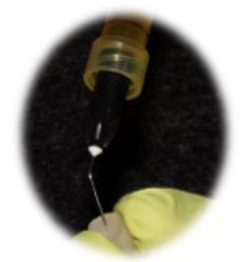

Ácido Fluorhídrico 20 seg

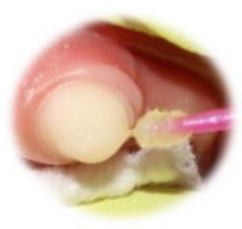

Adhesivo

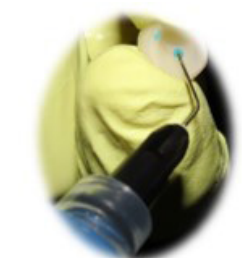

Ácido grabador $1 \mathrm{~min}$

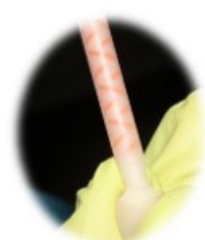

Cemento resinoso dual

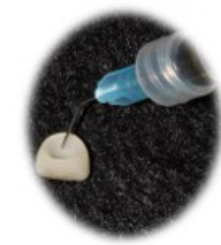

Silano $1 \mathrm{~min}$

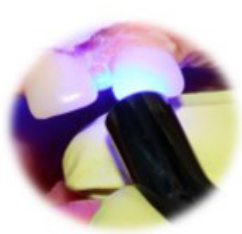

Fotocurado por 20 seg en cada cara

Figura 8: Tallado de dientes y cementado de coronas de disilicato de litio

Finalmente, se refirióal pacientea terapia de lenguaje quienes recomendaron ejercicios miofuncionales y de reposicionamiento de la lengua para estimular la función y fonación.

\section{Resultados}

Se obtuvieron resultados satisfactorios en el momento de concluir la rehabilitación bucal, mejorando la autoestima del paciente, se puede observar que en la fotografía de sonrisa inicial (Figura 1) presentaba una clasificación de "sonrisa Mona Lisa", caracterizada por la acción de los músculos cigomáticos mayores, moviendo las comisuras hacia afuera y arriba, seguido de una elevación gradual del labio superior, ${ }^{17}$ sin exposición dentaria, al contrario de la fotografía final de sonrisa (Figura 9) donde se mostró una sonrisa franca con exposición dental, resultando de la contracción de todos los músculos elevadores y depresores de los labios y de las comisuras. Se evidencia una mayor estética, su cara es más armónica, percibimos un aumento en el tercio inferior de la cara de $29,8 \%$ a $32,2 \%$; observándose un equilibrio de los tercios faciales $\mathrm{y}$
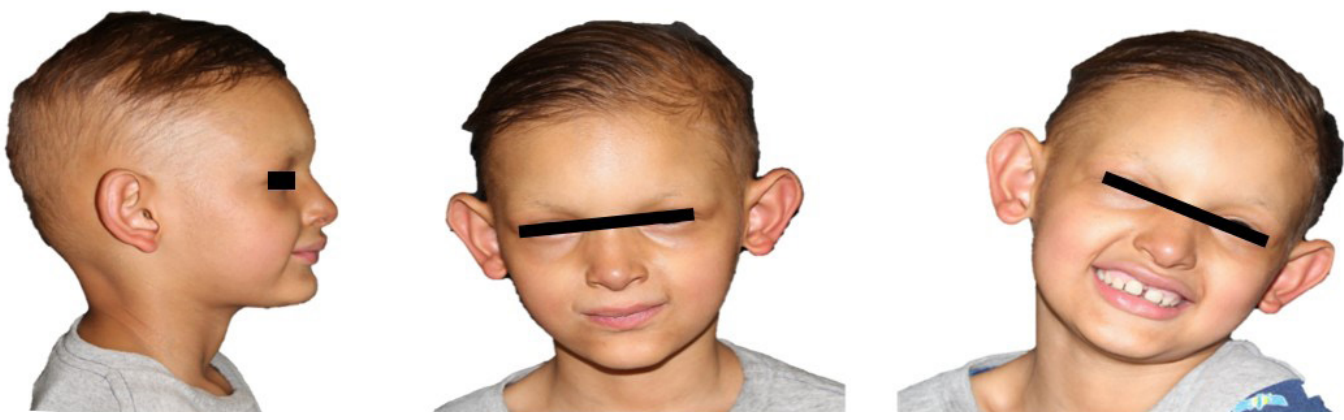

Figura 9: Fotografías extraorales finales 


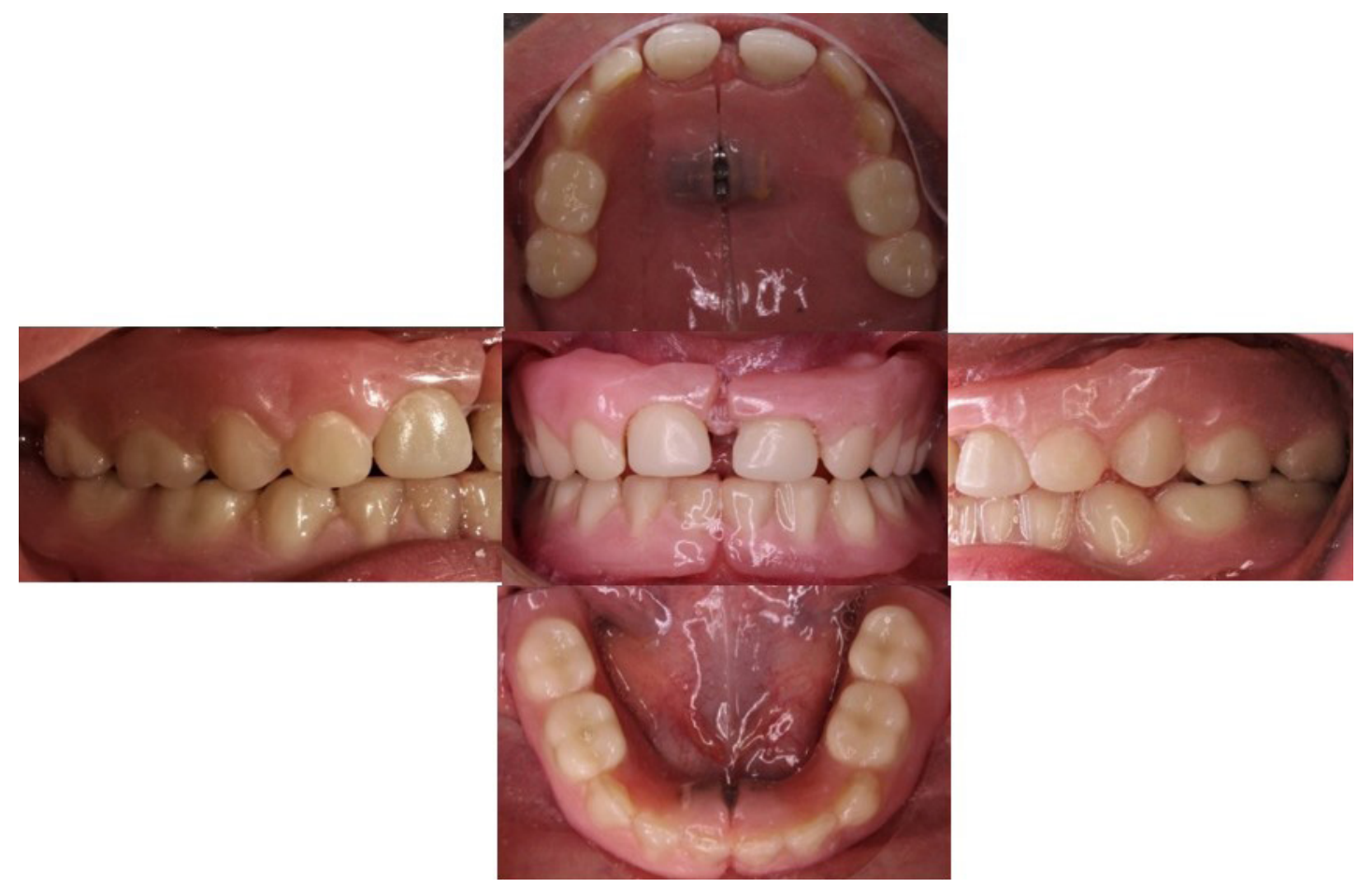

Figura 10: Fotografias intraorales finales

mejorando el perfil inicial superior en $3 \mathrm{~mm}$ y el inferior en $0 \mathrm{~mm}$ (Figura 9).

En el seguimiento a los 2 meses se observó una mejor adaptación y retención de las prótesis, debido a que el paciente refirió no necesitar la utilización de adhesivo (Ultra Corega de GSK); de igual manera, se evidenció una mejor función masticatoria debido al reporte de la madre que indica que el niño ya puede masticar diversos alimentos más duros como la manzana sin trocear. Por otra parte, hubo una mejoría en la fonación, debido a que anteriormente no pronunciaba casi ninguna palabra $y$ a los dos meses ya no tiene problemas en pronunciar palabras con las letras $\mathrm{T}$, $\mathrm{P}, \mathrm{C}, \mathrm{K}$ y mejorando la pronunciación de palabras con $G, R, S, F$, por lo que se recomienda continuar su proceso se terapia de lenguaje.

\section{Discusión}

La displasia ectodérmica (DE) incluye un gran número de trastornos hereditarios congénitos, los cuales se caracterizan por presentar una afección morfológica y funcional en los tejidos y derivados de la capa embrionaria ectodérmica, en los que hay dos o más estructuras involucradas, siendo las más afectadas el cabello, la piel, las glándulas sudoríparas, las uñas y los dientes. ${ }^{2,14,18-20}$

La displasia ectodérmica en la mayoría de sus casos va a presentarse debido a una expresión genética, pudiendo ser: autosómico dominante (OMIM\#129490), autosómico recesivo (OMIM\#224900) $\mathrm{o}$ recesivo ligado al cromosoma $X$ (OMIM\#305100). ${ }^{1,2,9}$ 
El tipo más común es debido a un gen recesivo ligado al cromosoma $X$, presente en la displasia ectodérmica hipohidrótica la cual presenta la triada de: hipotricosis, hipohidrosis e hipodoncia, siendo el $80 \%$ de los afectados. Este presenta una mutación del gen EDA1 que se asigna en el cromosoma Xq-12-q13.1; EDA1 sintetiza a la Ectodisplasina A, una proteína colagenosa implicada en la estructura ectodérmica y la osteogénesis. ${ }^{13,6,12,14,18,21}$

La displasia ectodérmica anhidrótica es autosómica dominante con la mutación del gen EDA1 y al igual que en la DEH va a presentar la triada de hipotricosis, oligodoncia y anhidrosis, siendo esta última la diferencia con la DEH. ${ }^{10,13,18}$

La displasia ectodérmica hidrótica se caracteriza por presentar hipotricosis, distrofia ungueal e hiperqueratosis de las palmas de las manos y plantas de los pies; se considera autosómica dominante por una mutación en el gen de la conexina, GJB6 o conexina-30. ${ }^{12,18}$

Las características físicas generales que pueden presentar estos pacientes son: cabello escaso, fino, hipopigmentado, quebradizo y de crecimiento lento, alteración en las glándulas sudoríparas, piel hipopigmentada, distrofia ungueal, alteración en las glándulas lagrimales, glándula tiroides, en el oído interno, disminución en la agudeza visual, hiperqueratosis de las palmas de las manos y plantas de los pies. En el caso del paciente, presenta diagnóstico de Displasia Ectodérmica Anhidrótica por lo que su principal característica es la anhidrosis, no presenta alteraciones en el oído interno, glándula tiroides, problemas visuales ni hiperqueratosis en manos y pies, por lo que debemos tener en cuenta que cada caso es diferente, algunos pacientes pueden tener características o alteraciones mínimas y otros tener afectados varios órganos y sistemas. ${ }^{1,3,4,14,22}$

Diversos autores mencionan que algunas de las características cráneo faciales que se pueden encontrar en estos pacientes son: frente y mentón prominente, pigmentación de la piel alrededor de los ojos y la boca, mayor distancia intercantal, implantación baja de las orejas, nariz en forma de silla de montar, biproquelia, depresión del tercio medio, dimensión vertical reducida, hipoplasia del maxilar, protrusión mandibular. En el presente caso podemos observar que el paciente presenta la mayoría de estas características menos la protrusión mandibular, por lo que al ser muy común que las presenten, se debe sospechar de una displasia ectodérmica si un paciente presenta dichas características y referirlo al genetista para el diagnóstico correspondiente. . $^{210,11,20}$

Es muy común que los pacientes con displasia ectodérmica presenten alteraciones orales, incluso puede ser uno de los principales parámetros para sospechar de algún síndrome o alteración, las anomalías más comunes que presentan son alteraciones de número dental como la hipodoncia u oligodoncia, alteraciones en la morfología dental como dientes cónicos, microdoncia o malformaciones dentales, alteraciones de estructura como la hipoplasia del esmalte, displasia alveolar, osteopenia, xerostomía y en algunos casos labio y paladar hendido. En el paciente observamos presencia de dientes cónicos únicos dientes presentes 
en boca, el 52 y 62, oligodoncia y displasia alveolar, es muy importante conocer las características de cada paciente para poder individualizar el plan de tratamiento del paciente. ${ }^{1,3,4,14}$

Cuando se rehabilita un paciente con displasia ectodérmica, es importante individualizar cada caso, debido a que pueden variar mucho las condiciones de cada paciente, la cantidad de dientes presentes, si existen alteraciones en su estructura, el colapso que existe en los maxilares, entre otros factores. El propósito del odontopediatra es restablecer la función del sistema estomatognático, la estabilidad, la fonación y la estética. ${ }^{4}$

La oligodoncia es definida como la ausencia de seis o más dientes, excluyendo los terceros molares. ${ }^{1} \mathrm{El}$ paciente presenta únicamente los dientes primarios $52 \mathrm{y}$ 62 presentando alteración de forma y en la ortopantomografía podemos observar la presencia de los dientes permanentes 11 y 21. Por lo que presenta oligodoncia de los dientes primarios 55, 54, 53, 51, $61,63,64,65,75,74,73,72,71,81,82$, $83,84,85$ y oligodoncia de los dientes permanentes: 17, 16, 15, 14, 13, 12, 22, 23, $24,25,26,27,37,36,35,34,33,32,31,41$, $42,43,44,45,46,47$, en el caso reportado se debe dar el diagnóstico de Anodoncia Parcial Verdadera. Urbina E, Santos A, Navarro E. $2017^{6}$ menciona un caso parecido al descrito en este reporte en el que dos pacientes de 12 y 19 años los cuales presentan agenesia de los dientes permanentes en ambas arcadas. Danelon M, Dalpasquale G, Gonzalez L. $2018^{18}$ observaron en una paciente de 5 años agenesia de los dientes 51,52, 61, 62, 63, $71,72,81,82,11,12,13,21,22,23,41,42$, 31,32 y alteración de forma de los dientes
53, 73 y 83. Ou-Yang L, Li T, Tsai A. $2019^{9}$ observaron en una paciente de 3 años la ausencia de incisivos y caninos superiores e inferiores.

Urbina E, Santos A, Navarro E. 20176, mencionan que una desventaja del uso de prótesis removibles es necesaria la cooperación del paciente y es muy importante el apoyo de los padres. En el presente caso no existió ningún problema en la cooperación por parte del paciente quien se sentía más confiado y feliz al utilizarlas, siendodemucha ayudaelinterés de la madre, que colaboró en mantener a su hijo motivado y comprometido; por lo que es muy importante comenzar la rehabilitación lo más pronto posible, para así generar resultados positivos en los pacientes.

La investigación de Ou-Yang L, Li T, Tsai A 2019, ${ }^{9}$ menciona que debido al poco desarrollo de los procesos alveolares, el uso de prótesis removibles es difícil, ya que se ve afectada su retención y estabilidad. En el presente caso reportado se evidenció una adecuada retención y estabilidad de las prótesis debido al uso continuo de las mismas que no requirió la utilización de adhesivos para ellas, pudiendo incluso morder manzanas enteras sin el desalojo de las prótesis, lo que anteriormente de su colocación no podía realizar.

El estudio de García J, Pulgar J. 2016, ${ }^{15}$ rehabilitaron protésicamente a pacientes pediátricos con oligodoncia, obteniéndose mayor armonía en los tejidos blandos de la cara, mayor bienestar emocional, estética, mayor eficiencia del sistema estomatognático, similares a los resultados del caso presentado en este trabajo. 
Es importante realizar un seguimiento de los pacientes pediátricos con oligodoncia debido a que se encuentran en constante crecimiento, generándose la necesidad de realizar los ajustes en las prótesis, por lo que se debería realizar un cambio de prótesis cuando el crecimiento de ambas arcadas lo requieran, y que muy probablemente debería ser alrededor del recambio de los dientes anterosuperiores. ${ }^{6}$ Los pacientes con Displasia Ectodérmica deben recibir un control odontológico según su riesgo de caries y ser más frecuente con de evitar o controlar sus desajustes, lo que adicionalmente permitiría evaluar la higiene bucal y flujo salival debido a la necesidad de preservar su salud bucal, lo que incidirá en su calidad de vida. relación al control de prótesis en virtud

pediatra, otorrinolaringólogo, psicólogo, foniatra, especialistas en estética $y$ oclusión, debido a que esta condición está asociada a oligodoncia y altera aparato estomatognático, así como, sus funciones primordiales como son la alimentación, fonación y estética.

El uso de coronas de disilicato de litio que requiere poco tallado de la estructura dental y el uso de prótesis parciales o totales removibles son opciones recomendadas en pacientes pediátricos con Displasia Ectodérmica para acompañar el crecimiento armónico de las estructuras bucales hasta el momento adecuado para realizar el tratamiento definitivo.

\section{Agradecimientos}

\section{Conclusiones}

Los pacientes con Displasia Ectodérmica deben ser tratados de forma multidisciplinaria por diversas especialidades médicas y odontológicas, entre ellas, el odontopediatra, genetista, pediatra, nutricionista o nutriólogo, ortodoncista, cirujano maxilofacial,
Los autores agradecemos la colaboración del alumno Leopoldo Pérez, la Lic. Edna Vilchis y la T.D. Carolina Díaz del Laboratorio de Prótesis Bucal Avanzada de la Escuela Nacional de Estudios Superiores, Unidad León, UNAM, México, por su ayuda en la elaboración de las prótesis removibles y coronas de disilicato de litio.

\section{Referencias bibliográficas}

1. Mascolo A, Boschetti E, Flanagan D. An ectodermal dysplasia patient treated with a small diameter implant supporting a single crown. Clin Cosmet Investig Dent. 2018;10:171-7.

2. Wang H, Hung K, Zhao K, Wang Y, Wang F, Wu Y. Anatomical analysis of zygomatic bone in ectodermal dysplasia patients with oligodontia. Clin Implant Dent Relat Res. 2019;21(2):310-6.

3. Correa MC, Salinas PA, Rico González GA, Arenas R. Anhidrotic ectodermal dysplasia: Report of 3 familial cases suggestive of X-linked inheritance. Dermatologia Cosmet Medica y Quir. 2014;12(3):175-8.

4. Kabilamurthi S, Ashok V, Ganapathy D. Various prosthetic treatment options for ectodermal dysplasia patients - A review. Drug Invent Today. 2019;12(6):1126-8.

5. Olivares JM, Hidalgo A, Pavez JP, Benadof D, Irribarra R. Functional and esthetic restorative treatment with preheated resins in a patient with ectodermic dysplasia : A clinical report. J Prosthet Dent [Internet]. 119(4):526-9. Available from: https://doi.org/10.1016/j.prosdent.2017.04.016

6. Urbina Vázquez E, Santos Espinoza A, Navarro Bori E. Tratamiento prostodóntico de pacientes con displasia ectodérmica hipohidrótica: reporte de dos casos. Rev Odontológica Mex [Internet]. 2017;21(1):49_ 53. Available from: http://dx.doi.org/10.1016/j.rodmex.2017.02.002 
7. Páez J, Correa C, González D, Rovira C. Manejo odontológico de pacientes con el síndrome de ChristSiemens-Touraine. Reporte de un caso. Rev Odontológica Mex [Internet]. 2018;22(1):51-5. Available from: http://www.scielo.org.mx/scielo.php?pid=S1870-199X2018000100051\&script=sci_arttext

8. Marín Botero ML, Espinal Botero G, Arroyo Fuentes TM, Posso Zapata M V., David Pérez M, Castañeda Peláez DA, et al. Displasia ectodérmica hipohidrótica: Reporte de casos. Av Odontoestomatol. 2013;29(1):11-23.

9. Ou-Yang LW, Li TY, Tsai AI. Early prosthodontic intervention on two three-year-old twin girls with ectodermal dysplasia. Eur J Paediatr Dent. 2019;20(2):139-42.

10. Cluzeau C, Hadj-Rabia S, Jambou M, Mansour S, Guigue P, Masmoudi S, et al. Only four genes (EDA1, EDAR, EDARADD, and WNT10A) account for $90 \%$ of hypohidrotic/anhidrotic ectodermal dysplasia cases. Hum Mutat. 2011;32(1):70-2.

11. Kilic S, Altintas SH, Yilmaz Altintas N, Ozkaynak O, Bayram M, Kusgoz A, et al. Six-Year Survival of a Mini Dental Implant-Retained Overdenture in a Child with Ectodermal Dysplasia. J Prosthodont. 2017;26(1):70-4.

12. Chappidi V, Voulligonda D, Bhogavaram B, Reddy PK. Ectodermal dysplasia: Report of two cases in a family and literature review. J Fam Med Prim Care [Internet]. 2017;6(2):169-70. Available from: http:// www.jfmpc.com/article.asp?issn=2249-4863;year=2017; volume=6;issue=1;spage=169; epage=170; aulast=Fa izi

13. Márquez M. Rehabilitación protésica de un niño de 3 años con displasia ectodérmica hipohidrótica. Rev OdontopediatríaLatinoam[Internet].2012;2(1):87-93.Availablefrom:https://www.revistaodontopediatria. org/ediciones/2012/1/art-10/

14. Schnabl D, Gerhard S, Biedermann R, Crismani A, Rasse M, Zauza K, et al. Dental Management and Prosthetic Rehabilitation of Patients Suffering from Hypohidrotic Ectodermal Dysplasia: A Report of Two Case Histories. Int J Prosthodont. 2018;31(6):552-7.

15. García A, Pulgar P. MANEJO IMPLANTOLÓGICO DE PACIENTE PEDIÁTRICO CON DISPLASIA ECTODÉRMICA: REPORTE DE CASO, SEGUIMIENTO A 10 AÑOS. acta Odont Venez. 2016;54.

16. Salazar-López C, Solar Q-D. Rehabilitación estética-funcional combinando coronas de disilicato de Litio en el sector anterior y coronas metal-cerámica en el sector posterior Aesthetic-functional rehabilitation combining Lithium disilicate crowns in the anterior region and me- tal-ce. Abr-Jun [Internet]. 2016;2626(22):102-9. Available from: http://www.scielo.org.pe/pdf/reh/v26n2/a07v26n2.pdf

17. Rubin L. The anatomy of a smile: its importance in the treatment of facial paralysis. Plast Reconstr Surg. 1974;53(4):384-7.

18. Danelon M, Dalpasquale G, Santos L, Garcia G, Carlos A, Delbem B. Displasia ectodérmica en odontopediatría. Rev Odontopediatría Latinoam [Internet]. 2018;8:75-82. Available from: https://www. revistaodontopediatria.org/ediciones/2018/1/art-8/

19. Dhamo B, Kuijpers MAR, Balk-Leurs I, Boxum C, Wolvius EB, Ongkosuwito EM. Disturbances of dental development distinguish patients with oligodontia-ectodermal dysplasia from isolated oligodontia. Orthod Craniofacial Res. 2018;21(1):48-56.

20. Wang Y, He J, Decker AM, Hu JC, Zou D. Clinical outcomes of implant therapy in ectodermal dysplasia patients: a systematic review. Int J Oral Maxillofac Surg. 2016;45(8):1035-43.

21. Güven Y, Bal E, Altunoglu U, Yücel E, Hadj-Rabia S, Koruyucu M, et al. Turkish Ectodermal Dysplasia Cohort: From Phenotype to Genotype in 17 Families. Cytogenet Genome Res. 2019;157(4):189-96.

22. Hsieh YL, Razzoog M, Hammaker SG. Oral care program for successful long-term full mouth habilitation of patients with hypohidrotic ectodermal dysplasia. Case Rep Dent. 2018;2018:11-3.

Recibido: $17 / 04 / 20$

Aceptado: 12/10/2020

Correspondencia: Jessica Vianey Aparicio, correo: vianey0401@gmail.com 